\title{
LA FORMULAZIONE DEL PILASTRO EUROPEO: QUIALI EFFETTI SUI DIRITTI SOCIALI?
}

THE FORMULATION OF THE EUROPEAN PILLAR. WHAT EFFECTS ON SOCIAL RIGHTS?

\section{CATERINA BUCCHERI}

Dottoranda dell'Istituto di Migrazioni

Università di Granata.

e-Revista Internacional de la Protección Social, ISNN 2445-3269. 2018, Vol. III, No 1 


\title{
RIASSUNTO
}

La garanzia dei diritti sociali e delle libertà fondamentali sono oggi una delle attività principali dell'U.E Solo negli anni '90 la Comunità Economica Europea ha iniziato a trasformarsi in Unione Europea, estendendo la sfera delle proprie attività dal campo economico a quello politico-sociale e l'ultima fase è stata la proclamazione del Pilastro Europeo dei Diritti Sociali.

Il pilastro non è legalmente vincolante, non implica automaticamente che i principi e $\mathrm{i}$ diritti contenuti in esso siano garantiti, considerando che la politica monetaria dell'Unione e la frattura delle diverse politiche nazionali dividono l'Europa. Per cambiare questo contesto si rende necessaria la conformazione in senso federale dell'istituzione europea. Tale istituzione dovrebbe avere personalità politica capace di aggregare e cambiare la politica monetaria, rivedendo la rigidità del Fiscal Compact, poiché ad oggi non tiene conto delle variabili economiche e sociali che l'Europa sta vivendo. Il successivo passo è l'introduzione di una politica sociale e di integrazione gestita direttamente dall'entità centrale, capace di unire, introducendo la solidarietà transnazionale, che sicuramente incentiverebbe la crescita di questo modello politico inclusivo.

PAROLE CHIAVE: Unione Europea. Diritti sociali. Pilastro sociale. Realizzazione. Impegno politico.

\begin{abstract}
The guarantee of social rights and fundamental freedoms are today one of the main activities of the E.U. Only in the 1990s did the European Economic Community begin to transform itself into the European Union, extending the sphere of its activities from the economic to the political and social fields. The last phase was the proclamation of the European Pillar of Social Rights.

The pillar is not legally binding; it does not automatically imply that the principles and rights contained are guaranteed, considering the monetary policy of the Union and the breakdown of the policies that divide Europe. To counter this path, it is necessary to conform the federal institution of a European institution that has a political personality capable of aggregating and changing monetary policy, revising the rigidity of the Fiscal Compact, as it does not take into account the economic and social variables that Europe is living. The next step is the introduction of a social and integration policy managed directly by the central entity, which manages to aggregate by introducing transnational solidarity, which would certainly encourage the growth of this inclusive political model.
\end{abstract}

KEYWORDS: European Union. Social rights. Social Pillar. Realization. Political Endeavor.

Questa comunicazione è stata presentata al Seminario Dottorale di Investigazione "Pilar Europeo de derechos sociales, trabajo y migraciones: nuevos retos" tenutosi il 4 e 5 aprile 2018 nell'ambito del Programma di Dottorato in Studi Migratori dell'Università di Granata.

e-Revista Internacional de la Protección Social, ISNN 2445-3269. 2018, Vol. III, No 1

http://dx.doi.org/10.12795/e-RIPS.2018.i02.12. Páginas: 215-229.

Página 216 
SOMMARIO

I. INTRODUZIONE

II. DIRITTI SOCIALI: L'ARTICOLATO PERCORSO DELL'UNIONE EUROPEA

III. INCIDENZA DEL PILASTRO NELLE POLITICHE SOCIALI DELL'UNIONE EUROPEA

IV. LA COMPLESSITÀ DEI SISTEMI WELFARE NAZIONALI NELL'ATTUAZIONE DEL PILASTRO EUROPEO

V. CONCLUSIONI

e-Revista Internacional de la Protección Social, ISNN 2445-3269. 2018, Vol. III, No 1 http://dx.doi.org/10.12795/e-RIPS.2018.i02.12. Páginas: 215-229. 


\section{INTRODUZIONE}

Il "modello sociale europeo" si fonda su un principio di natura generalista poiché ogni Stato membro adotta uno specifico prototipo di politiche sociali per rispondere alle esigenze dei propri cittadini, da cui deriva che la tutela dei medesimi diritti sociali avviene mediante forme legislative differenti. Va considerato che l'attuazione dello stato sociale richiede notevoli costi per la sua applicazione, pertanto l'uniformazione delle politiche sociali in Europa è un obiettivo difficilmente attuabile.

Il rispetto dei restrittivi parametri della Politica Monetaria Europea non ha consentito di rimuovere le disuguaglianze nell'area UE, sarebbe quindi necessario adottare un modello sociale uniforme per tutele e garanzie in tutti gli Stati membri.

Con la proclamazione del Pilastro europeo dei Diritti Sociali del 2015 la U.E. ha voluto assegnare agli stati membri una guida per migliorare le condizioni di vita dei cittadini. Da questo documento, però, non si evidenzia nulla per quanto attiene ai progetti relativi all'immigrazione ed ai processi di integrazione, che invece erano previsti dalla Carta dei Diritti Fondamentali dell’UE del 2001.

Tale lacuna rappresenta una discriminazione, considerato che sono state abbandonate le strategie di ammissione ed integrazione, la cui attuazione è lasciata ai singoli Stati membri, evidenziando i limiti politici della UE; la conseguenza è stata una mancanza di garanzia dei diritti, che se anche enunciati con standard comunitari, sono risultati limitati e non possono rappresentare un riferimento per un auspicato modello generale da applicare nell'Unione.

Nell'ambito dei paesi membri si osservano quattro tipi diversi di modello di stato sociale, da distinguere tra quelli applicati negli Stati meridionali, in quelli anglosassoni, negli Stati del nord e negli Stati continentali, che saranno successivamente approfonditi.

Soltanto una trasformazione politica in senso federale dell'U.E. potrà realmente eliminare le disuguaglianze sociali con una politica di sostegno economico all'esercizio dei diritti sociali, avocando a se le risorse e la distribuzione delle stesse al fine di ottenerne una effettiva fruibilità.

Dopo la crisi economica del 2008 gli Stati membri, soprattutto quelli con maggior debito nazionale, hanno diminuito le risorse destinate allo stato sociale, e siccome la materia economica è sovranazionale, con l'imposizione del rispetto dei parametri monetari, nei singoli stati le fasce deboli, tra cui gli immigrati stranieri, hanno visto progressivamente diminuire le loro garanzie sociali, dimostrando che la sola unione monetaria è insufficiente a fronteggiare il divario sociale oggi presente nell'area UE Difatti, nei singoli Stati membri, la capacità economica del welfare, è l' unico parametro che rende concreto ed efficace il godimento dei diritti sociali.

\section{DIRITTI SOCIALI: L'ARTICOLATO PERCORSO DELL'UNIONE EUROPEA}

La tutela dei diritti umani e le libertà essenziali rappresentano oggi una delle attività più importanti dell'Unione Europea che in questo campo è recente, poiché solo negli anni

e-Revista Internacional de la Protección Social, ISNN 2445-3269. 2018, Vol. III, No 1

http://dx.doi.org/10.12795/e-RIPS.2018.i02.12. Páginas: 215-229.

Página 218 
Novanta la Comunità Economica Europea ha iniziato a trasformarsi in Unione Europea, estendendo la sfera delle sue attività dal campo economico a quello politico-sociale.

I Trattati Europei si limitavano ad affermare che i diritti delle persone dovevano essere tenuti in considerazione dall'Unione, nell'elaborazione delle proprie politiche, come principi guida ma senza l'efficacia giuridica di una norma ${ }^{1}$.

L'evoluzione dei diritti sociali in ambito UE ha subito un percorso lungo e tortuoso.

Con l'istituzione della C.E.E. nel 1957, l'art. 117 stabiliva che le politiche sociali erano di esclusiva competenza degli Stati membri e con l'art. 118 si attribuiva alla Commissione soltanto una facoltà limitata al coordinamento della collaborazione tra gli stessi in materia di diritti sociali.

Col Trattato di Parigi del 1951, con l'istituzione della C.E.C.A. (Comunità Europea del Carbone e Acciaio) ne fu costituita la Corte di Giustizia che con il Trattato di Roma del 1957 divenne la Corte di Giustizia delle Comunità Europee (C.G.C.E.).

Tale organismo, con la propria giurisprudenza è stato il primo passo della U.E. per contribuire e consolidare i diritti sociali nel quadro normativo europeo, rendendo tali principi generali idonei a far parte dell'ordinamento europeo. Col Trattato di Roma, successivamente modificato con l'Atto Unico Europeo del 1987, si enunciano espressamente alcune competenze comunitarie in materia di diritti sociali. Tra gli altri, vengono dichiarati i principi per la libera circolazione dei lavoratori e della parità salariale tra uomo e donna.

La protezione dei diritti sociali in ambito U.E. venne sancita nel 1989 nel Consiglio europeo di Strasburgo, con l'adozione della Carta comunitaria dei diritti sociali fondamentali dei lavoratori, che comunque lasciava agli Stati membri la competenza di attuarli. Si trattava, quindi, di un atto pragmatico che ne dettava i principi.

Le competenze del settore sociale vengono ampliate con l'introduzione del Trattato di Maastricht nel 1992, dove viene sancito il perseguimento dell'occupazione e della protezione sociale, il miglioramento della qualità della vita, la coesione economicasociale e la solidarietà tra gli stati membri.

Nel 1997 il trattato di Amsterdam riconosce costituzionalmente i diritti sociali quale criterio direttivo della politica sociale della UE.

Nel 2000 viene votata dal Consiglio Europeo di Nizza la Carta dei Diritti Fondamentali dell'U.E, un documento che elenca con precisione l'insieme dei diritti di cui cittadini e residenti dell'Unione sono beneficiari.

Ma la svolta rilevante si ha nel 2007, quando con l'adozione del trattato di Lisbona, la Carta cessa di essere una mera dichiarazione di principi, ed assume valore giuridico vincolante. Quest'ultima esprime le libertà e i diritti nei quali l'Unione riconosce il proprio patrimonio giuridico ed è attinente alla dignità, alla libertà, all'uguaglianza, alla

\footnotetext{
${ }^{1}$ Gradilone, S, 'L'attenzione in merito alla tutela dei diritti umani e delle libertà fondamentali nell'Unione Europea", 2017.Recuperato nell'aprile 2018 dal sito: https://www.balcanicaucaso.org/aree/Europa/Unione-europea-una-questione-di-diritti-177090.
}

e-Revista Internacional de la Protección Social, ISNN 2445-3269. 2018, Vol. III, Nº 1 
solidarietà, alla cittadinanza e alla giustizia, il cui rispetto è già tutelato dai principi costituzionali comuni agli stati membri e dalla Convenzione Europea per la Salvaguardia dei Diritti dell'Uomo e delle Libertà Fondamentali².

Un altro passo è compiuto il 17 novembre 2017, in occasione del vertice sociale europeo di Göteborg, quando viene proclamato ufficialmente il Pilastro Europeo dei Diritti Sociali (European Pillar of Social Rights), dichiarato nell'anno 2015 dal Presidente Juncker nel discorso sullo Stato dell’Unione.

Secondo la Unione Europea il Pilastro tiene conto delle trasformazioni in atto nel mondo dell'occupazione e rappresenterà la guida per un rinnovato processo di convergenza verso migliori condizioni di lavoro e di vita tra gli Stati membri, attraverso un equo e buon funzionamento dei mercati del lavoro e dei sistemi di welfare ${ }^{3}(3)$.

Il Pilastro europeo mira a rendere più efficaci i diritti per i cittadini e si basa su 20 principi chiave, strutturati in tre categorie quali le pari opportunità e accesso al mercato del lavoro, condizioni di lavoro eque e protezione sociale e inclusione.

E' sostenuto da un quadro di valutazione di indicatori chiave e serve da riferimento per monitorare il "progresso sociale", con cui si intende la capacità di favorire e sostenere il benessere e le opportunità, creando condizioni che consentano alle persone di sviluppare appieno il loro potenziale e di soddisfare le loro necessità di base.; tale strumento di monitoraggio permetterebbe di vagliare i risultati occupazionali e sociali ottenuti dagli Stati membri con la media dell'UE e della zona euro e in alcuni casi anche con quelli di altri attori internazionali e di compierne un'analisi comparativa. L'insieme delle valutazioni verrebbe discusso con i comitati competenti del Consiglio, in vista dell'inserimento nella relazione annuale comune sull'occupazione, pubblicata ogni autunno nell'ambito dei Semestri europei.

La gamma dei temi affrontati è molto ampia in ragione dell'articolazione e della complessità sociale ma anche per la diversità delle condizioni in cui vivono i cittadini della UE I principi danno gli orientamenti per affrontare i nuovi sviluppi nel mondo del lavoro e nella società al fine di realizzare un'economia sociale di mercato competitiva che mira alla piena occupazione e al progresso sociale.

Si tratta di settori per lo più di competenza politica degli Stati membri e di ambito nazionale. Ma all'Unione Europea viene affidato il compito di definire il quadro

\footnotetext{
${ }^{2}$ La Convenzione europea per la salvaguardia dei diritti dell'uomo e delle libertà fondamentali o CEDU è una Convenzione internazionale redatta e adottata nell'ambito del Consiglio d'Europa, firmata a Roma il 4 novembre 1950 e integrata e modificata da 14 Protocolli aggiuntivi. La CEDU è considerata il testo centrale in materia di protezione dei diritti fondamentali dell'uomo perché è l'unico dotato di un meccanismo giurisdizionale permanente che consenta a ogni individuo di richiedere la tutela dei diritti ivi garantiti, attraverso il ricorso alla Corte europea dei diritti dell'uomo, con sede a Strasburgo.

${ }^{3}$ Pilastro Europeo dei Diritti Sociali: Il Pilastro è stato presentato in due forme giuridiche di identico contenuto: una raccomandazione della Commissione, che ha efficacia a partire da subito, e una proposta di proclamazione congiunta del Parlamento, del Consiglio e della Commissione. Su tali basi la Commissione avvierà le discussioni con il Parlamento Europeo e il Consiglio per assicurare al Pilastro un ampio sostegno politico e l'approvazione ad alto livello.
}

e-Revista Internacional de la Protección Social, ISNN 2445-3269. 2018, Vol. III, N 1

http://dx.doi.org/10.12795/e-RIPS.2018.i02.12. Páginas: 215-229.

Página 220 
generale di tali diritti e la direzione da intraprendere per renderli effettivi. La stessa ha inoltre una parte di competenze proprie per agire in modo da dare attuazione ai principi enunciati.

L'idea alla base del Pilastro è quella di creare un quadro di riferimento con indicatori specifici al fine di favorire la convergenza sociale tra gli Stati membri dell'Unione e intervenire, attraverso la creazione di nuovi strumenti, là dove l'integrazione all'interno dell'Unione Economica e Monetaria aveva creato conflitti negativi.

La proclamazione del Pilastro Europeo è un chiaro segnale di svolta nel discorso europeo sui diritti sociali, un'opportunità di cambiamento che, se efficacemente sfruttato, potrebbe innescare dinamiche positive al momento inattese. Rappresenta il primo ed essenziale passo per l'avvio di un'Europa più sociale anche se la sua implementazione richiede l'azione non solo delle istituzioni europee, ma anche dei governi, dei partner sociali degli Stati Membri e delle rispettive amministrazioni federali e regionali ${ }^{4}$.

Obiettivo del Pilastro è dare concretezza alle disposizioni della Carta dei diritti fondamentali dell'Unione europea, considerandolo così strategicamente importante da richiedere una procedura originale e non ordinaria. La Carta migliora il "corpus giuridico" e garantisce alcuni diritti personali, civili, politici, economici e sociali dei cittadini e dei residenti dell'Unione, con particolare riferimento al principio di non discriminazione nelle sue diverse manifestazioni.

Per l'attuazione del P.E.D.S. la Commissione indica l'adozione di un accordo interistituzionale da parte del Parlamento e del Consiglio che costituirebbe un atto solenne diretto a valorizzare, attraverso l'impegno condiviso delle varie istituzioni europee, la sua cruciale natura.

Rimane però il rischio di inconsistenza di tale iniziativa nonché l'incapacità di produrre effetti concreti nel breve-medio periodo, finendo di fatto con l'accendere speranze poi disattese, poiché l'iter legislativo dovrebbe essere sostenuto da tutti gli Stati membri.

I contenuti del Pilastro sono legati all'occupazione e, per come sono formulati rischiano di tradursi in diritti sociali intesi come diritti collettivi, conferiti in base allo stato di occupazione; i diritti sociali sono invece diritti individuali applicabili a tutti, indipendentemente dalla posizione lavorativa.

Il Pilastro è uno strumento per riconoscere e affrontare i cambiamenti in atto a livello sociale, demografico, economico, professionale. Intende creare i presupposti e accompagnare il cammino dei Paesi UE verso una confluenza in tale ambito, così che ogni cittadino dell'Unione abbia eguali diritti, tutele e opportunità. Dopo anni di impegno mirati all'integrazione del mercato unico e della moneta,vuole portarel'attenzione sui cittadini e sulla realtà sociale,auspicando un più stretto coordinamento delle politiche sociali e del lavoro anche attraverso cooperazioni rafforzate tra paesi membri con l'estensione di maggiori tutele. ${ }^{4}$ Menéndez-Valdés J., E.U. Social Pillar: “A Potential New Start For Europe. Social Europe”. Londra,
Gran Bretagna. 2017. Recuperato nell’ aprile 2018 dal sito: https://www.socialeurope.eu/50326-

e-Revista Internacional de la Protección Social, ISNN 2445-3269. 2018, Vol. III, No 1

http://dx.doi.org/10.12795/e-RIPS.2018.i02.12. Páginas: 215-229.

Página 221 


\section{INCIDENZA DEL PILASTRO NELLE POLITICHE SOCIALI DELL'UNIONE EUROPEA}

Il Pilastro europeo presenta delle criticità riguardanti in primis la sua natura giuridica che, come atto di proclamazione, non è idoneo a promuovere una effettiva rifondazione politico-costituzionale dei diritti sociali, considerandoli come strumenti di attivazione sul mercato del lavoro, a scapito della dimensione protettivo- redistributiva.

Al Pilastro, è applicato il sistema del "metodo aperto di coordinamento", dove i singoli stati sono orientati a raggiungere diversi obiettivi comuni seguendo però strade diverse, con il monitoraggio dell'Unione.

Si tratta di un metodo che prevede un sistema volontario di cooperazione e confronto politico, degli obiettivi comuni da raggiungere e presume la collaborazione della società civile e delle parti sociali.

Un limite rappresentato da questo metodo è la mancanza di integrazione della politica sociale con le altre politiche comunitarie, specialmente con quella monetaria, ad oggi l'unica raggiunta, nella quale la UE può esercitare il controllo e applicare sanzioni a tutti gli Stati membri; in ambito di politiche sociali però, non ha ancora degli strumenti definiti per l'uniformazione e l'applicazione delle stesse, pertanto non può svolgere una funzione di controllo ma solo limitarsi all'osservazione.

Le strutture politiche della UE considerano l'unione economica e monetaria come un progetto sociale avente come finalità la creazione di condizioni favorevoli ad una crescita forte e condivisa per tutti i cittadini appartenenti all'Unione. Ma nella realtà, i costi delle politiche sociali e le restrizioni in materia monetaria continuano a far permanere negli Stati membri una situazione di disparità sociale poiché gli stati meno virtuosi, hanno difficoltà ad estendere i benefici a tutta la popolazione.

Risulta inattuabile una politica sociale comunitaria che possa essere applicata in egual modo a tutti gli Stati membri, tenuti a rispettare i parametri monetari, specialmente se tra questi non tutti hanno uguali condizioni economiche. Sebbene il Pilastro Europeo dei Diritti Sociali debba rappresentare un quadro di riferimento affinché determinati diritti sociali possano essere non solo sanciti ma resi localmente attuabili, non è accompagnato da alcuna direttiva che consenta agli Stati in sofferenza economica di derogare ai patti monetari.

Dare valore al Pilastro rientra tra le priorità politiche delineate dall'UE per costruire un'Unione più inclusiva e più equa, attraverso la lotta contro la povertà, la ricostruzione del capitale umano, la capacità di recupero dagli shock asimmetrici e l'importanza dell'economia sociale. I suddetti obiettivi saranno perseguiti in relazione e nel quadro della revisione di medio termine della Strategia Europa 2020, che dovrebbe portare ad un processo decisionale più inclusivo, basato sul coinvolgimento delle parti sociali, sul coordinamento delle politiche economiche, occupazionali e sociali che va ulteriormente rafforzato in conformità con le procedure esistenti e nel pieno rispetto delle competenze nazionali. Inoltre, considerato il massiccio flusso immigratorio nell'area UE, non è ragionevole pensare di attuare i diritti sociali senza considerare l'apporto economico e sociale reso dagli immigrati stranieri extracomunitari.

e-Revista Internacional de la Protección Social, ISNN 2445-3269. 2018, Vol. III, No 1

http://dx.doi.org/10.12795/e-RIPS.2018.i02.12. Páginas: 215-229.

Página 222 
Infatti, mentre nella Carta era prevista la predisposizione di un progetto in materia di immigrazione che includesse le procedure di ammissione, sottolineando l'adozione di misure di integrazione dei cittadini di paesi terzi, auspicando un quadro comune europeo con principi fondamentali ${ }^{5}$, di questo progetto nel Pilastro non si trova riscontro, a conferma che l'UE non avendo la competenza esclusiva in materia sociale, non riesce a renderne giuridicamente efficaci i diritti. Inoltre, ciò risulta manchevole poiché manifesta una disparità basata sulla condizione amministrativa dello straniero non UE, in quanto a livello nazionale "le norme sull' immigrazione e sul trattamento dello straniero precludono l'accesso al godimento paritario dei diritti, anche di quelli fondamentali" $"$

Malgrado sia stato riconosciuto il diritto alla migrazione, continuano a persistere barriere che ogni stato membro della UE ha elevato all'esercizio del medesimo diritto.

Nell'ottica del controllo dell'immigrazione irregolare infatti, gli Stati attuano un progressivo restringimento dello stesso diritto di emigrare.

L'immigrazione continua a rimanere una questione che gli stati vogliono riservare alla loro azione politica ed alla sovranità nazionale, che rappresenta il limite oltre il quale la UE non riesce a proseguire. Se l'immigrato straniero extracomunitario gode per legge dei diritti in misura inferiore ed è soggetto a doveri più onerosi rispetto ai cittadini comunitari, si continuerà ad avere un grave problema di discriminazione da sanare attraverso la riforma delle disposizioni di legge, oppure per mezzo della giurisdizione costituzionale.

Sebbene con la proclamazione di Göteborg si è tentato di fornire un quadro di riferimento condiviso a livello sovranazionale, si deve auspicare una guida che conduca ad una concreta attuazione del progetto coinvolgendo i Governi degli Stati membri. Per porre in atto il Pilastro è necessario progettare misure concrete e strumenti che lo sorreggano; se ciò non venisse attuato sarebbe disattesa, ancora una volta l'istituzione Europea.

Il Pilastro non contiene espliciti riferimenti ai cittadini stranieri non comunitari, ed infatti il Governo Italiano ${ }^{7}$ il 22 dicembre 2017 ha inviato alla Commissione europea un riscontro ufficiale dell'Italia alla consultazione. Nella sua osservazione, il conseguimento degli obiettivi descritti del Pilastro europeo possono essere conseguiti sia mediante l'espansione di adeguate politiche sociali a livello nazionale, sia con la realizzazione partecipata di strumenti ed azioni a livello UE.

\footnotetext{
${ }^{5}$ Chiaromonte, W., Lavoro e diritti sociali degli stanieri, Torino, Italia. Giappicchelli Editore 2013

${ }^{6}$ Favilli, G., I diritti dell'immigrato non regolarmente soggiornante, L'Aquila, Italia: Edizione Il Sirente. 2008, P. 295

${ }^{7}$ Governo italiano: nell'Ordinamento giuridico italiano il Governo esercita il potere esecutivo. Secondo l'art. 92 c. 1 della Costituzione si distinguono tre diversi organi: il presidente del Consiglio dei ministri (primo ministro), i ministri e il Consiglio dei ministri. La formazione del Governo è disciplinata in modo succinto dagli art. 92, c. 2, 93 e 94 della Costituzione e da prassi costituzionali consolidatesi nel tempo.

Il governo dipende dalla fiducia di entrambi i rami del parlamento ed ha in suo potere la possibilità di emettere decreti legge solo in caso di emergenza.
}

e-Revista Internacional de la Protección Social, ISNN 2445-3269. 2018, Vol. III, N 1

http://dx.doi.org/10.12795/e-RIPS.2018.i02.12. Páginas: 215-229.

Página 223 
L'integrazione dei cittadini immigrati stranieri extracomunitari richiede un approccio integrato e sistematico che guardi a tutte le dimensioni della vita, con particolare attenzione alla scuola, al lavoro, alla sanità ed alla previdenza ${ }^{8}$.

Non è previsto un intervento normativo solo da parte delle istituzioni europee, ma un insieme di azioni svolte dagli organismi competenti nel rispetto dei principi di sussidiarietà e di proporzionalità, assicurando così che l'attuazione possa rispettare le differenze nazionali e locali, così marcate all'interno della UE. D'altronde non si tratta di introdurre una legislazione del tutto nuova, ma di integrare quanto già esiste nell'acquis comunitario ${ }^{9}$ e di renderne effettive o ampliarne le tutele là dove è necessario.

Altro obiettivo del Pilastro è il bilanciamento degli squilibri economici all'interno dell'Unione Economica e Monetaria ${ }^{10}$, esasperate durante la crisi finanziaria del 2008, che si vorrebbe attuare mediante le Politiche del Lavoro, ovvero nel dare una risposta ai divari che si sono venuti a creare tra i paesi aderenti alla moneta unica, a causa dell'imposizione di regole comuni.

I parametri di Maastricht rappresentano un modello di sviluppo economico differente tra i paesi del Nord dell'UE maggiormente favoriti dai criteri dell'UEM e quelli del Sud più svantaggiati, a causa degli squilibri di bilancio che devono fronteggiare, che si aggravano maggiormente quando si trovano a fronteggiare, come singolo Stato, emergenze di natura umanitaria.

\footnotetext{
${ }^{8}$ Governo Italiano, 2017. Recuperato nel april 2018 de: http://www.governo.it /approfondimenti /2017/12/pilastro-europeo-dei-diritti-sociali-alla-camera-lesame-del-testo/.

${ }^{9}$ L'acquis di Schengen è un insieme di norme e disposizioni, integrate nel diritto dell'Unione europea, volte a favorire la libera circolazione dei cittadini all'interno del cosiddetto Spazio Schengen, regolando i rapporti tra gli Stati che hanno siglato la Convenzione di Schengen. Il complesso di norme, dette anche accordi di Schengen, prende il nome dalla cittadina di Schengen, in Lussemburgo. L'acquis di Schengen comprende l'Accordo di Schengen, firmato il 14 giugno 1985 dagli Stati del Benelux, dalla Germania e dalla Francia, la Convenzione di applicazione dell'Accordo di Schengen, firmata il 19 giugno 1990, gli accordi di adesione alla Convenzione di applicazione dell'Accordo di Schengen da parte di Italia, Spagna, Portogallo, Grecia, Austria, Danimarca, Finlandia e Svezia.

I nuovi paesi membri UE dal 2004 e dal 2007 sono obbligati a entrare nello spazio Schengen, ma per quattro di essi l'accordo non è ancora entrato in vigore: gli altri paesi UE hanno ottenuto un periodo transitorio prima di avviare la libera circolazione delle persone, inoltre i nuovi paesi membri devono dotarsi di tutte le infrastrutture adeguate a implementare il sistema di informazione Schengen.

${ }^{10}$ L'Unione economica e monetaria (U.E.M) dell'Unione europea è stata sancita con il Trattato di Maastricht, che, attraverso tre successive fasi, concluse un lungo processo di diplomazia il cui esito è più notoriamente rappresentato dal conio e adozione di una moneta unica europea, l'euro, in sostituzione delle rispettive valute nazionali da parte dei 19 paesi (Austria, Belgio, Cipro, Estonia, Francia Germania, Grecia, Irlanda, Italia, Lettonia, Lituania, Lussemburgo, Malta, Paesi Bassi, Portogallo, Slovacchia, Slovenia e Spagna) con una politica monetaria comune sotto il controllo della Banca Centrale Europea (BCE), dando vita alla cosiddetta eurozona. Le politiche economiche di ciascuno stato sono coordinate tra loro attraverso l'Unione, perciò rimangono ancora prerogative degli Stati che però accettano una limitazione posta dal Trattato sul funzionamento dell'Unione Europea (TFUE).
}

e-Revista Internacional de la Protección Social, ISNN 2445-3269. 2018, Vol. III, N 1

http://dx.doi.org/10.12795/e-RIPS.2018.i02.12. Páginas: 215-229.

Página 224 


\section{LA COMPLESSITÀ DEI SISTEMI WELFARE NAZIONALI NELL'ATTUAZIONE DEL PILASTRO EUROPEO}

Nell'attuazione del Pilastro si evidenzia un problema di tipo politico, cioè l'indisponibilità a modificare le regole europee da parte di quei paesi che, fino ad oggi, ne hanno maggiormente beneficiato.

Il Patto di stabilità e crescita (PSC) è un accordo firmato nel 1997 dai paesi membri dell'Unione Europea, collegato al controllo delle politiche di bilancio pubbliche, con l'obiettivo di conservare i requisiti di adesione all'Unione economica e monetaria dell'Unione Europea (Eurozona) consolidando il cammino d'integrazione monetaria iniziato nel 1992 con il Trattato di Maastricht. Il rispetto del PSC non permette ai Governi alcun margine alla propria politica di bilancio, e quindi diviene inattuabile l'eventuale applicazione del Pilastro, che cambierebbe strutturalmente il Welfare, senza modificare la politica economica della U.E ${ }^{11}$.

Inoltre le scelte dell'Unione in materia di Governance economica, con ricadute sulle decisioni dei singoli stati membri in materia di spesa pubblica per il sociale, devono essere considerate in funzione dell'assenza di competenze in materia sociale dell'UE, riferendosi alle competenze dirette, le quali, comunque, difficilmente saranno concesse all'Unione in futuro, poiché per fare ciò, servirebbe l'unanimità degli stati membri che, al momento, è difficile da raggiungere e continua a rappresentare uno dei grandi limiti dell'Unione.

Al fine di rendere efficace il Pilastro, l'obiettivo della UE è quello di poter valutare la conformità degli ordinamenti degli stati membri, rispetto alle disposizioni del testo, tenendo presente che le raccomandazioni della Commissione non sono legalmente vincolanti e non hanno alcuna efficacia giuridica, esse fungono soltanto da dichiarazioni di principio che dovrebbero ispirare le future scelte politiche degli stati UE.

La pubblicazione della raccomandazione non è la tappa conclusiva del lavoro sul Pilastro sociale. Con la sua approvazione, la Commissione ha preso posizione, aprendo così un'ulteriore fase di confronto con il Consiglio e il Parlamento europeo in vista dell'adozione di un testo condiviso, oltre a proseguire il dialogo con la società civile sugli orientamenti adottati.

Intanto la Commissione ha già avviato l'iter di approvazione di alcuni provvedimenti in tema di equilibrio tra attività professionale e vita familiare, informazione ai lavoratori $\mathrm{e}$ accesso alla protezione sociale.

Questo approccio implica una chiara convergenza di visioni e di interventi da parte di tutti i soggetti coinvolti e costituisce una scommessa importante per il futuro della costruzione europea, essendo in gioco la capacità di collaborare.

I principi individuati nel pilastro intendono accompagnare e guidare la trasformazione del lavoro a seguito della globalizzazione, della rivoluzione digitale e dell'affermarsi di

\footnotetext{
${ }^{11}$ Corti, F. y Vesan, P, "Il pilastro europeo dei diritti social". 2018. Recuperado nel april 2018 de: http://www.secondowelfare.it/primo-welfare/innovazione-sociale/il-pilastro-europeo-dei-diritti-sociali-
}

e-Revista Internacional de la Protección Social, ISNN 2445-3269. 2018, Vol. III, No 1

http://dx.doi.org/10.12795/e-RIPS.2018.i02.12. Páginas: 215-229.

Página 225 
nuovi modelli organizzativi. Non si dimenticano poi le sfide del calo demografico e dei cambiamenti sociali in corso.

Il progresso economico e sociale sono tra loro connessi e l'espansione di un Pilastro Europeo dei diritti sociali deve essere parte di un'applicazione volta a realizzare un modello di crescita più inclusivo e sostenibile, rendendo migliore la competitività dell'Europa e presentandola più favorevole agli investimenti, alla realizzazione di posti di lavoro, al consolidamento della unione sociale senza abbandonare l'elemento immigrazione, che oggi è parte integrante dell'UE, e di cui si continua a trascurarne l'aspetto economico e sociale ${ }^{12}$.

I progetti volti alla realizzazione del modello di sviluppo impegneranno sia l'UE. che gli Stati membri; con il mercato unico, i dispositivi finanziari e gli strumenti della politica saranno impiegati per l'eliminazione degli ostacoli. Come precedenza immediata, la Commissione ha precisato le misure da scegliere indicando una strategia di uscita attendibile, riformando il sistema finanziario, assicurando il ristabilimento del bilancio finalizzato alla crescita a lungo termine e rafforzando l'organizzazione con l'Unione economica e monetaria. Per raggiungere risultati concreti servirà una Governance economica più forte che sappia armonizzare le priorità, gli obiettivi principali e i rapporti sui singoli paesi che sosterranno gli Stati membri a programmare le proprie strategie per ristabilire la sostenibilità della crescita e delle finanze pubbliche. A livello dell'UE saranno ammesse direzioni integrate che rivestano le priorità e i traguardi dell'Unione, mentre agli Stati membri verranno rivolte raccomandazioni specifiche $^{13}(13)$.

Il pilastro europeo dei diritti sociali mira a fungere da guida per realizzare risultati sociali e occupazionali efficaci in risposta alle sfide attuali e future così da soddisfare $\mathrm{i}$ bisogni essenziali della popolazione e garantire una migliore attuazione e applicazione dei diritti sociali.

L'UE spinge per la cooperazione tra le due componenti della democrazia rappresentativa europea, così come declinata nell'art. 10 T.U.E ${ }^{14}$.

Auspica il rilancio di una politica della solidarietà in Europa mediante il Pilastro dei diritti sociali che viene espressamente considerato come parte integrante del processo di convergenza all'interno dell'Unione economica e monetaria e che, pur non essendo destinato ad ampliare le competenze dell'Unione, tende a valorizzare quelle espressamente già riconosciute e a rafforzare l'azione degli stati membri nel garantire una maggiore attenzione a questo versante del processo di integrazione ${ }^{15}$.

\footnotetext{
${ }^{12}$ Sabbattini, G, "La Governance unitaria dell'area euro, pilastro della crescita inclusiva dell'Unione Europea”, Revista Manifesto Sardo, no. 23/2017.

${ }^{13}$ Pierini, M., Crisi e ripresa economica in Europa. 2014, Artículo recuperado en april de 2018 de: http://www.europadirectmarche.it/notiziario/2014

${ }^{14}$ Il Trattato di Maastricht, o Trattato dell'Unione europea (TUE), è un trattato che è stato firmato il 7 febbraio 1992 a Maastricht nei Paesi Bassi, dai dodici paesi membri dell'allora Comunità Europea, oggi Unione europea, che fissa le regole politiche e i parametri economici e sociali necessari per l'ingresso dei vari Stati aderenti nella suddetta Unione. È entrato in vigore il $1^{\circ}$ novembre 1993.

${ }^{15}$ Saitto, F.; "Risocializzare l'Europa. La dimensione sociale europea tra economia e integrazione sovranazionale", 2018. Articolo recuperato nell'aprile 2018 dalla rivista http://www.diritticomparati.it /rivista/risocializzare-leuropa-la-dimensione-sociale-europea-tra-economia-di-mercato-e-integrazionesovranazionale/
}

e-Revista Internacional de la Protección Social, ISNN 2445-3269. 2018, Vol. III, No 1

http://dx.doi.org/10.12795/e-RIPS.2018.i02.12. Páginas: 215-229.

Página 226 
L'impegno sul fronte del Pilastro dei diritti sociali dovrà tenere conto del rilievo che in questo ambito continuano a rivestire le scelte sovrane in materia welfaristica dei singoli stati membri.

Questo impegno politico collega strutturalmente democrazia e solidarietà nell'instaurazione di un processo di convergenza, che deve portare a ricostruire la fiducia verso un'Unione europea che sia realmente in grado di rispondere alle domande sociali che l'attuale fase pone.

Ciò al fine di promuoverne un nuovo concetto che tenga conto del contesto storico in cui gli Stati sono chiamati ad operare, provando in tal modo a rilanciare il processo di integrazione europea.

Inoltre la UE dovrebbe tenere conto che attualmente non esiste un unico modello sociale applicato da tutti gli Stati membri, ma che tali modelli si diversificano all'interno degli stessi a seconda dei percorsi storici degli Stati.

Nella UE vengono individuati quattro modelli teorici di welfare, il modello socialdemocratico dei paesi del nord Europa, il modello liberale degli Stati anglosassoni, il modello corporativo degli stati continentali e il modello mediterraneo degli stati meridionali. Essi sono derivati dai differenti percorsi storici ed economici degli Stati e differiscono principalmente per il tipo di prestazione erogata e ai finanziamenti previsti.

Il modello nord-Europa è caratterizzato dal principio dell'universalismo, ha come obiettivo la protezione di tutti, si basa sullo stato di bisogno di ogni individuo e riconosce socialmente l'uguaglianza dei cittadini. A differenza, il modello liberale è finalizzato alla riduzione della povertà, gli interventi sono mirati a specifici gruppi dirischio ed il mercato del lavoro è il principale mezzo per ammortizzare la povertà. Il modello corporativo si orienta alla protezione dei lavoratori e delle loro famiglie quando la capacità delle stesse di provvedere ai bisogni dei componenti diviene insufficiente. Gli interventi statali riducono la dipendenza dal mercato del lavoro; nel welfare mediterraneo è la famiglia che deve assicurare il soddisfacimento dei bisogni ai suoi componenti e lo Stato svolge un ruolo marginale, non fornendo alcun tipo di sussidio economico ma soltanto erogando servizi. Ciò determina un processo di regionalizzazione delle politiche sociali che danno origine ad un divario tra le parti più ricche di un paese con quelle maggiormente svantaggiate, creando situazioni di discriminazione sociale.

Vagliando i sistemi sociali si osserva che quelli nord-europei e continentali raggiungono obiettivi nella media indicata dalla UE. Mentre il sistema anglosassone raggiunge appena il livello medio di soddisfacimento europeo, quello mediterraneo in funzione dei limitati benefici prima della pensione, non raggiunge, per alcuni paesi tra i quali l'Italia, la soglia minima sociale dell'U.E ${ }^{16}$.

\footnotetext{
${ }^{16}$ Borger, V. (2013), "How the Debt Crisis Exposes the Development of Solidarity in the Euro Area, in European Constitutional Law Review", (pag. 9: 7-36, 74). Articolo pubblicato sulla rivista Cambridge University Press (nr. 9) il 19.04.2013
}

e-Revista Internacional de la Protección Social, ISNN 2445-3269. 2018, Vol. III, N 1

http://dx.doi.org/10.12795/e-RIPS.2018.i02.12. Páginas: 215-229.

Página 227 
La distorsione evidente è che la UE dovrebbe porre maggiore attenzione al capitale e non solo al lavoro, come fino ad oggi attuato, e questa politica col passare del tempo, in assenza di correttivi economici, aumenterà il divario sociale tra gli Stati U.E., nonostante il buon proposito di attenzionare $\mathrm{i}$ diritti, in ultimo, la proclamazione del P.S.D.E.

Il Pilastro, così come la politica di integrazione adottata dalla UE, mostra i suoi limiti considerando il lavoro come un'integrazione di fatto. Esso è valutato quale punto di arrivo del percorso integrativo dello straniero immigrato, che dovrebbe creare la propria vita sociale intorno allo stesso.

Ma si tratta soltanto di un'integrazione economica, che non porta con se alcun beneficio sociale, relegando gli immigrati stranieri extracomunitari ai margini della società, così come successo in Francia, in occasione delle rivolte nelle Banlieue.

E' auspicabile che la UE indirizzi il Pilastro nella direzione dell'evoluzione di un modello di integrazione che contempli alcuni cardini applicabili in tutta l'Unione, quali la lingua del paese che accoglie, la cultura, l'educazione scolastica e il diritto alla sanità, che fino ad oggi sono regolati in maniera generica.

Con l'impostazione che l'Unione vuole dare al Pilastro, rendendo centrale il ruolo del lavoro, si crea un contesto in cui è l'immigrato straniero non comunitario a doversi accostare alla società civile. Invece è quest'ultima a dover cambiare il proprio sistema di contatto, dirigendosi verso la realtà dell'immigrazione straniera per ottenere benefici reciproci.

Per fare questo, è necessario mantenere alta l'attenzione sul Pilastro e sulla sua Proclamazione, che le parti sociali, i partiti politici e l'opinione pubblica si mobilitino per ricordare che la sottoscrizione della Proclamazione inter-istituzionale è un impegno politico strategico. Certamente, non è un atto legalmente vincolante e la sua sottoscrizione non implica automaticamente che i principi e diritti in esso contenuti siano garantiti, per questola volontà politica sarà determinante per attuare iniziative legislative concrete rendendo più difficile ai governi venire meno all'impegno sottoscritto a Göteborg.

\section{CONCLUSIONI}

Obiettivo del Pilastro è attuare l'integrazione attraverso le politiche del lavoro poiché, secondo l'istituzione europea esso rende possibili le relazioni sociali. Ma se fosse applicato nella sua odierna struttura originerebbe soltanto un'integrazione economica, e non offrirebbe alcun vantaggio sociale, relegando gli immigrati stranieri non comunitari ai margini della società.

Le parti sociali, i partiti politici e l'opinione pubblica devono considerare l'attuazione del pilastro come un impegno politico strategico fondamentale ma, vista la sua natura non giuridicamente vincolante, non implica automaticamente che i principi e i diritti in esso contenuti siano garantiti, considerati i rilevanti costi da affrontare per l'attuazione delle politiche sociali e il rigore finanziario imposto dalla politica monetaria dell'Unione.

e-Revista Internacional de la Protección Social, ISNN 2445-3269. 2018, Vol. III, No 1

http://dx.doi.org/10.12795/e-RIPS.2018.i02.12. Páginas: 215-229.

Página 228 
A causa della rigidità dell'UEM, al fine di ridurre gli squilibri economici e sociali tra gli Stati membri, la UE si è focalizzata sulla mobilità dei lavoratori, ma la mancanza di una unione politica e fiscale ha evidenziato che tutti gli atti, compreso il P.E.D.S., si rivolgono a sistemi politici eterogenei e che non si finalizzano con la solidarietà transnazionale, rendendo di fatto impraticabile un sistema di welfare comune. Prova ne è l'invito del 2013, in piena crisi economica, della Germania e di altri Stati nord europei alle istituzioni comunitarie al fine di rivedere le regole sulla mobilità dei cittadini U.E. ed extracomunitari in senso restrittivo, finalizzato ad evitare l'abuso del welfare del paese ospitante. Sebbene contrario ai principi della libera circolazione questo invito ha ispirato nel 2015 la proposta della Commissione del Labour Mobility Package, che mirava all'introduzione di criteri stringenti per l'accesso al welfare del paese cheaccoglie, ma, ad oggi non ha condotto ad alcun atto direttivo in tale senso.

Pertanto la U.E., che ha fondato la propria idea di integrazione e socialità sull'efficienza del mercato del lavoro, per aumentare la competitività e sanare gli squilibri, col Pilastro vorrebbe garantire a tutti $\mathrm{i}$ cittadini, in ambito comunitarioe, residualmente agli immigrati extracomunitari, condizioni di parità con i cittadini del paese ospitante. Ma così come la politica monetaria, fondata sulla mobilità del lavoro, mostra ad oggi un'Europa economicamente divisa, ragionevolmente anche le politiche sociali, se realizzate con l'attuale testo del Pilastro, a medio termine, mostreranno i limiti e l'incapacità di integrare ed unire.

La percezione è che gli Stati membri con economie avanzate si ritengono attorniati dagli immigrati economici e ciò li induce a ritoccare verso il basso il loro sistema sociale con l'introduzione di correttivi legati alla tipologia ed alla durata dei contratti di lavoro ${ }^{17}$.

Per poter garantire eguali diritti sociali la UE deve dare slancio alle politiche di investimento nelle aeree meno efficienti, invertendo la tendenza della mobilità del lavoro, aprendo la sua politica agli immigrati extracomunitari che troverebbero spazio a pieno titolo nello stato sociale e rappresenterebbero una ricchezza economica e culturale.

L'attuale frattura delle politiche economiche che dividono l'Europa può essere sanata solo dalla costituzione di un'istituzione che abbia personalità politica capace di aggregare e dal cambio della politica monetaria, rivedendo la rigidità del Fiscal Compact, che deve essere reso maggiormente flessibile, poiché ad oggi non tiene conto delle variabili economiche e sociali che l'Europa sta vivendo.

E' necessario che congiuntamente all'istituzione di uno Stato comune europeo si affianchi una politica sociale e di integrazione gestita direttamente dall'entità centrale, che riesca ad aggregare introducendo la solidarietà transnazionale e che sicuramente incentiverebbe la crescita di questo modello politico inclusivo.

\footnotetext{
${ }^{17}$ Direttiva nr. 2004/38. (2004). Art. 7, par. 1, lett. b.; art. 12, co. 2; art. 13 co. 2 e art. 14 co. 1 , che subordinano la libertà di circolazione e soggiorno dei cittadini comunitari inattivi al non costituire un «onere eccessivo» per il sistema di assistenza sociale dello Stato membro ospitante.
}

e-Revista Internacional de la Protección Social, ISNN 2445-3269. 2018, Vol. III, No 1

http://dx.doi.org/10.12795/e-RIPS.2018.i02.12. Páginas: 215-229.

Página 229 